
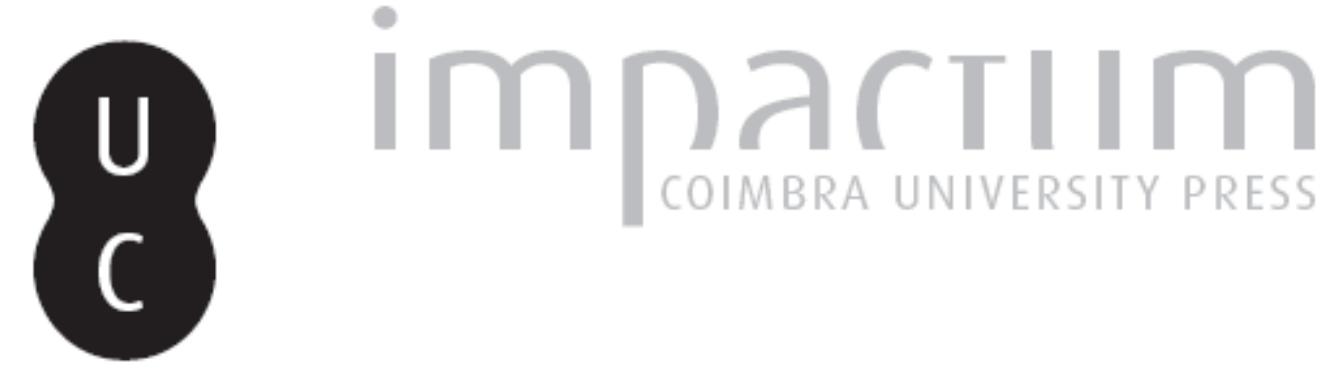

\title{
Empresas estrangeiras e capital humano nos serviços intensivos em conhecimento
}

Autor(es): $\quad$ Rêgo, Helena Maria Ferreira; Varum, Celeste Amorim; Carneiro, Anabela

Publicado por: Faculdade de Economia da Universidade de Coimbra

URL persistente:

URI:http://hdl.handle.net/10316.2/25226

DOI:

DOI:http://dx.doi.org/10.14195/2183-203X_32_1

Accessed : $\quad$ 26-Apr-2023 12:47:28

A navegação consulta e descarregamento dos títulos inseridos nas Bibliotecas Digitais UC Digitalis, UC Pombalina e UC Impactum, pressupõem a aceitação plena e sem reservas dos Termos e Condições de Uso destas Bibliotecas Digitais, disponíveis em https://digitalis.uc.pt/pt-pt/termos.

Conforme exposto nos referidos Termos e Condições de Uso, o descarregamento de títulos de acesso restrito requer uma licença válida de autorização devendo o utilizador aceder ao(s) documento(s) a partir de um endereço de IP da instituição detentora da supramencionada licença.

Ao utilizador é apenas permitido o descarregamento para uso pessoal, pelo que o emprego do(s) título(s) descarregado(s) para outro fim, designadamente comercial, carece de autorização do respetivo autor ou editor da obra.

Na medida em que todas as obras da UC Digitalis se encontram protegidas pelo Código do Direito de Autor e Direitos Conexos e demais legislação aplicável, toda a cópia, parcial ou total, deste documento, nos casos em que é legalmente admitida, deverá conter ou fazer-se acompanhar por este aviso.

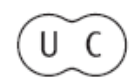




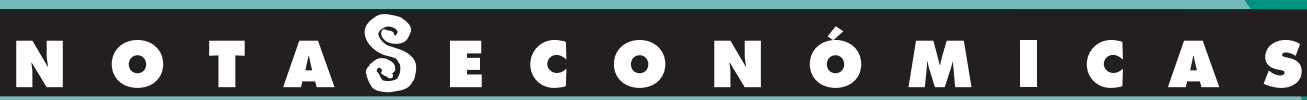

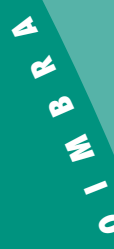

HELENA RÊGO / CELESTE AMORIM VARUM /

ANABELA CARNEIRO

EMPRESAS ESTRANGEIRAS E CAPITAL HUMANO NOS SERVIÇOS INTENSIVOS EM CONHECIMENTO

JOÃO GABRIEL FIDALGO / MIND THE GAP: EDUCATION INEQUALITY AT THE REGIONAL LEVEL IN PORTUGAL, MARTA SIMÕES / ADELAIDE DUARTE

MARCO M. SORGE $1986-2005$

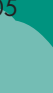

ON THE EMPIRICAL SEPARABILITY OF NEWS SHOCKS AND SUNSPOTS

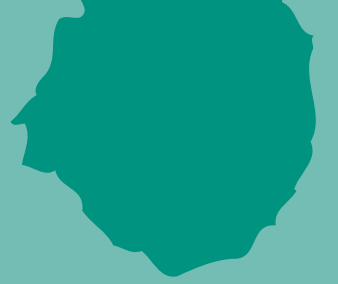


Empresas Estrangeiras e Capital Humano nos Serviços Intensivos em Conhecimento*

O efeito das empresas estrangeiras na economia receptora poderá ocorrer através do mercado de trabalho, contribuindo aquelas para o desenvolvimento do capital humano. Este artigo investiga se as empresas estrangeiras contribuíram positivamente para o desenvolvimento do capital humano, analisando o sector dos serviços intensivos em conhecimento em Portugal. $A$ análise empírica baseia-se num painel de empresas no período de 2000 a 2006 construido com dados dos Quadros de Pessoal. Verifica-se que as empresas com participação estrangeira no capital social apresentam, tudo o resto constante, uma maior intensidade de capital humano do que as empresas nacionais.
L'effet des entreprises étrangères sur l'économie du pays d'implantation peut se produire via le marché du travail en contribuant au développement du capital humain de l'économie d'implantation. Cet article étudie la contribution positive des entreprises étrangères au développement du capital humain, en analysant le secteur des sociétes de services spécialisées dans la connaissance (les «Knowledge Intensive Business Services») au Portugal. L'étude se base sur un panel de données au niveau des entreprises pour la période de 2000 à 2006. La base de données utilisée est Quadros de Pessoal. Les résultats mettent en évidence que les entreprises ayant un capital étranger présentent, un capital humain plus qualifié que les entreprises nationales

The effect of foreign firms on host economies may occur through labour market, leading to the development of human capital. This paper investigates whether foreign firms contributed in a positive way to the development of human capital, looking in detail at the knowledge intensive services in Portugal. The study is based on panel data at the firm level for the period 2000-2006 using the Quadros de Pessoal dataset. The results show that firms with foreign capital have higher human capital intensity than domestic firms.

\footnotetext{
* Agradecemos ao Gabinete de Estratégia e Planeamento (GEP) do Ministério do Trabalho e Solidariedade Social (MTSS) por gentilmente ter facultado o acesso à base de dados dos Quadros de Pessoal. O tratamento dos dados, resultados e conclusões são da exclusiva responsabilidade dos autores. Agradecemos ainda a um revisor anónimo pelos comentários pertinentes.

† Agradece o suporte da Fundação para a Ciência e Tecnologia (FCT) [SFRH/S=BSAB/ 920/ 2009].
} 
As multinacionais tornaram-se nas últimas décadas agentes económicos de grande destaque, centrais no processo de crescimento e modernização das economias. O efeito das multinacionais ocorre, por um lado, pela transferência de tecnologia, contribuindo para um upgrade tecnológico da economia receptora (Blomström e Kokko, 1998; Baldwin et al., 2005). O seu efeito poderá ocorrer também de forma indirecta, nomeadamente através do mercado de trabalho, contribuindo aquelas para o desenvolvimento do capital humano.

A literatura na temática (ex. Slaughter, 2002 e Slaughter e Blonigen, 2001; Lunati and O'Connor 1999; Blomström e Kokko, 1998; 2003; Larudee e Koechlin, 1999; Lipsey, 2004; Kapstein, 2002) revela que a relação entre capital humano e investimento directo estrangeiro é bidireccional ${ }^{1}$. Por um lado, o nível de capital humano é um factor de atracção de Investimento Directo Estrangeiro (IDE). Por outro lado, a presença estrangeira pode contribuir para o desenvolvimento do capital humano.

Este artigo analisa a hipótese de as empresas estrangeiras contribuírem positivamente para o desenvolvimento do capital humano. Especificamente, o artigo testa se as empresas estrangeiras apresentam, comparativamente com as nacionais, maior intensidade de capital humano, medido pela percentagem de trabalhadores que detêm determinados níveis de qualificações ou habilitações, na linha do adoptado, por exemplo, por Bouquet et al. (2004). Este artigo incide sobre o sector dos serviços intensivos em conhecimento (SIC), que tem sido descurado pela literatura nesta temática, apesar da sua importância em termos de produto, emprego e investimento (UNCTAD, 2004).

O sector dos serviços intensivos em conhecimento é composto por empresas que vendem serviços para outras empresas em que o conhecimento (trabalho qualificado, e conhecimento incorporado quer nas pessoas quer em tecnologia) e a informação são os principais componentes. O conhecimento é utilizado para satisfazer uma necessidade específica do cliente (Miles, 2005; Tomlinson, 2002).

Em 2005, os SIC representavam 33,3\% do emprego na Europa (EU-25). Entre 2000 e 2005 os serviços de alta intensidade tecnológica cresceram a uma taxa anual de $2,7 \%$, e Portugal $(9,9 \%)$ foi dos países que registou maiores taxas de crescimento (EUROSTAT, 2007). Assistimos também a um crescimento significativo do investimento directo estrangeiro neste sector (EUROSTAT, 2004). O crescimento do outsourcing, a procura crescente pelas tecnologias de informação (TIC) criaram a oportunidade para o aparecimento de serviços especializados. Por último, algumas mudanças que ocorreram no mercado de trabalho, em particular o aparecimento de certo tipo de qualificações escassas e que são melhor remuneradas nos SIC, e o facto de um número cada vez maior de pessoas preferir trabalhar em actividades que proporcionam maiores oportunidades de aprendizagem (European Foundation, 2005), têm contribuído para o crescimento deste sector.

O sector engloba um conjunto de actividades determinantes no crescimento e competitividade das economias e regiões (Miles et al., 1996; Tomlinson, 2002; Miles, 2005; Harrington e Daniels, 2006; Bishop, 2008). Os serviços aí incluídos são tidos como elementos fundamentais na criação e comercialização de novos produtos e novos processos e desenvolvimento do capital humano (Tomlinson, 2002; OECD, 2006; Quevedo e Verdú, 2008).

O estudo empírico é aplicado à economia portuguesa utilizando a base de dados dos Quadros de Pessoal, extremamente representativa do sector privado da economia portuguesa (Almeida, 2004). No contexto dos SIC, procura-se testar as seguintes hipóteses:

10 Investimento directo estrangeiro define-se por ser um investimento de longo prazo em que o investidor estrangeiro tem uma influência dominante na gestão da empresa (UNCTAD, 2005). De acordo com a OCDE (1999) para que esta influência exista o investidor precisa de deter pelo menos $10 \%$ do capital social da empresa. 
$\left.1^{\circ} .^{\circ}\right)$ Se as empresas estrangeiras possuem uma maior intensidade de capital humano face às suas congéneres nacionais;

$\left.2^{\circ} .^{\circ}\right)$ Se a percentagem que o investidor estrangeiro detém no capital social é relevante em termos de comportamento diferenciador no que respeita à intensidade do capital humano.

$\mathrm{O}$ artigo encontra-se estruturado em quatro partes. Na segunda parte procede-se ao enquadramento teórico sobre a relação IDE, sector de serviços intensivos em conhecimento e desenvolvimento do capital humano. Os resultados empíricos são apresentados na terceira secção. Por último, na quarta secção apresentam-se os principais contributos e conclusões.

\section{IDE, SIC e Capital Humano}

De acordo com a UNCTAD (2009), o peso dos serviços no stock mundial de investimento directo estrangeiro (IDE) passou de 49\% em 1990 para 64\% em 2007, apresentando o IDE neste sector uma taxa de crescimento superior à de outros sectores. Existem três razões que explicam este crescimento. A primeira tem a ver com o crescimento da importância dos serviços em termos de emprego e de valor acrescentado. A segunda razão tem a ver com o carácter não transaccionável da maior parte dos serviços, o que significa que precisam de ser produzidos e consumidos no mesmo local (Bouquet et al., 2004). A terceira razão tem a ver com a liberalização das políticas em relação ao IDE nos serviços face ao reconhecimento dos seus efeitos positivos, nomeadamente no acesso a tecnologias mais avançadas (Blomström e Kokko, 2003; Wang et al., 2002; EUROSTAT, 2004).

Apesar da significativa presença estrangeira no sector dos serviços, e deste nas economias, a grande maioria dos estudos que analisa os efeitos do IDE sobre o capital humano foca na manufactura. Apesar de algumas especificidades, estes estudos permitem-nos compreender a forma como o IDE neste sector poderá contribuir para o desenvolvimento do capital humano (Boddewyn et al., 1986; Dunning, 1989).

Uma das primeiras condições para a existência de IDE consiste na existência de falhas de mercado. Para que as empresas realizem IDE estas devem possuir algumas vantagens próprias que lhes permitam competir com sucesso num ambiente hostil (Hymer, 1976; Dunning, 1977). As vantagens das empresas estrangeiras face às domésticas podem decorrer de conhecimentos (como capital humano, tecnologia, vantagens organizacionais) ou estar relacionadas com a dimensão e capacidade produtiva. Nos serviços, estas vantagens são maioritariamente elementos intangíveis (Bouquet et al. 2004; UNCTAD, 2004), centradas no conhecimento (por exemplo sobre clientes, padrões de consumo, software e hardware), capacidade e organização das actividades, economias de escala e gama. A internacionalização via IDE permite às empresas explorar as suas vantagens internacionalmente da melhor forma, ao reduzir a probabilidade de spillover do conhecimento e os custos de transacção, protegendo ao mesmo tempo a qualidade do produto/serviço prestado e tornando possível um maior conhecimento do mercado (Boddewyn et al., 1986; Dunning, 1989).

A capacidade de uma região atrair IDE nos serviços está intimamente relacionada com a dimensão do mercado e a liberalização, boa rede de infra-estruturas de transporte e de comunicação e informação, assim como também com a disponibilidade de recursos (instituições e recursos humanos) qualificados. Também, ou ainda mais, nos serviços, a relação entre capital humano e IDE é bidireccional (Kapstein, 2002). Por um lado, o nível de capital humano é um factor de atracção de IDE. Por outro lado, a presença estrangeira pode contribuir para o desenvolvimento do capital humano (Blomström e Kokko, 2003; Gorg e Eric, 2005).

Os efeitos da presença estrangeira no mercado de trabalho podem ser analisados no âmbito de um modelo concorrencial de procura e oferta de trabalho qualificado, modelo em que as curvas da procura e oferta têm as inclinações habituais (Slaughter e Blonigen, 2001) 2 . 
Neste artigo consideramos a influência da presença estrangeira ao nível da procura de trabalho qualificado. Essa influência pode ocorrer de três formas. A primeira resulta da transferência das vantagens específicas da multinacional (tecnologia em forma de capital ou conhecimento) para as filiais, a qual poderá implicar procura de trabalhadores qualificados para a utilização eficaz dessa tecnologia (Slaughter, 2002). A segunda é através dos efeitos spillovers. Os conhecimentos que as empresas filiais receberam da empresa mãe podem difundir-se pela economia receptora, pelo que vão ser necessários mais trabalhadores qualificados nas empresas que por contágio começam a utilizar essa tecnologia. Por último, a presença estrangeira pode contribuir para o aumento da procura de trabalho qualificado através dos investimentos em capital (Slaughter e Blonigen, 2001). O aumento do capital físico está associado a economias de escala que, para serem aproveitadas, necessitam de mercado com alguma dimensão. Para o conseguirem, precisam, por vezes, de políticas de marketing e de publicidade avançadas e trabalhadores devidamente qualificados para as levarem a cabo (Lunati e O'Connor, 1999).

Entre outros, os estudos de Wang et al. (2002), Narula e Marin (2003), Blomström e Kokko (2003), Barbosa (2007) e Siegel et al. (2005) revelam a maior intensidade de trabalhadores qualificados entre empresas estrangeiras. Outros estudos levantam algumas dúvidas sobre a relação, pois apesar de demonstrarem uma maior intensidade de capital humano, as multinacionais podem não ter um efeito significativo sobre a procura de trabalhadores qualificados. Por exemplo, Almeida (2004) revela que existiu um efeito selecção na década de 90 em Portugal, que o autor designou de efeito cherry pick up; os investidores estrangeiros adquiriram empresas nacionais que já possuíam trabalhadores qualificados.

As motivações dos investidores determinam, em grande medida, o tipo de procura de mão-deobra por parte das empresas estrangeiras. Os investimentos orientados para a exploração de recursos naturais são intensivos em capital, necessitando de pouco trabalho qualificado (Te Velde, 2005). Se o objectivo do investimento for explorar a dimensão do mercado ou evitar custos de transporte elevados, ou porque é importante estar perto do cliente (Bergman, 2006), as filiais investem pouco na qualificação da mão-de-obra e em formação. Já no investimento orientado para a racionalização de custos, a existência de mão-de-obra barata e não qualificada é um factor importante (Te Velde e Morrissey, 2001, 2004). Por fim, o investimento motivado pela procura de activos estratégicos, caracteriza-se pela criação de filiais que usam tecnologias avançadas e desenvolvem actividades de inovação. Como tal, é de esperar que seja o tipo de investimento em que haja mais procura por trabalhadores qualificados e investimento em formação (Te Velde, 2005).

Para além das motivações dos investidores, outros factores influenciam o efeito do IDE sobre o emprego. Existem diferenças entre sectores, sendo de esperar que o IDE nos serviços tenha maior efeito sobre o emprego qualificado (Blomström e Kokko, 2003; UNCTAD, 2004). Esta suposição decorre de dois aspectos concretos. Enquanto que na indústria é possível a divisão do processo produtivo em fases, separando-se geograficamente as que necessitam de trabalho menos qualificado das que necessitam de trabalho mais qualificado, nos serviços esta separação é mais difícil. Por outro lado, no IDE nos serviços predomina a transferência de tecnologia no sentido de conhecimento (Boddewyn et al., 1986; Bouquet et al., 2004; UNCTAD, 2004), muitas vezes associado à utilização de equipamento sofisticado, pelo que a experiência $e$ habilitações dos trabalhadores (conjugados com a implementação de programas de educação e formação específica) são factores importantes para o sucesso da transferência das práticas (UNCTAD, 2004). Com base na revisão de literatura exposta, espera-se que as empresas estrangeiras nos sectores intensivos em conhecimento tenham um comportamento diferenciado em termos de capital humano, isto é, que empreguem uma força de trabalho com mais habilitações e qualificações. 


\section{Análise Empírica}

\section{1. $A$ base de dados}

Os dados utilizados neste estudo são oriundos da base de dados dos Quadros de Pessoal, que contém informação sobre empresas, estabelecimentos e trabalhadores para a quase totalidade dos sectores da economia portuguesa desde 1982.

Com base no número de identificação das empresas, construiu-se uma base de dados em painel para as empresas pertencentes ao sector dos SIC, para o período de 2000 a 2006 (excepto 2001) ${ }^{3}$. A amostra inclui 306413 observações a que corresponde, em média, cerca de 51068 empresas por ano.

Seguindo a classificação do EUROSTAT, o sector SIC engloba actividades com elevada intensidade tecnológica (medida pelas despesas de I\&D); actividades fortemente utilizadoras dessa tecnologia; actividades intensivas em conhecimento (medida pelas qualificações dos trabalhadores); e as actividades de educação, saúde ${ }^{4}$, as actividades recreativas e de lazer e alguns serviços de transporte. Nesta linha, o sector dos serviços encontra-se dividido em dois grandes grupos: serviços intensivos em conhecimento e serviços com menor intensidade de conhecimento.

Como se pode observar pela leitura da Tabela 1, o peso dos SIC no total dos sectores tem vindo a crescer ao longo do período considerado, quer em termos de número de empresas, emprego ou volume de vendas. Em 2006 eram responsáveis por 26\% do emprego total no sector privado da economia Portuguesa e por $25 \%$ do volume total de vendas.

\begin{tabular}{|c|c|c|c|}
\hline & 2000 & 2003 & 2006 \\
\hline \multicolumn{4}{|c|}{ Serviços intensivos em conhecimento } \\
\hline N. ${ }^{\circ}$ empresas & 31617 & 50003 & 63975 \\
\hline Emprego & 561733 & 646070 & 806237 \\
\hline Volume de vendas* & 36047,18 & 67985,97 & 68595,60 \\
\hline Vendas médias por empresa & 1,14 & 1,40 & 1,07 \\
\hline \multicolumn{4}{|c|}{ Todos os sectores } \\
\hline N. ${ }^{\circ}$ empresas & 266853 & 305843 & 343953 \\
\hline Emprego & 2699609 & 2848286 & 3099513 \\
\hline Volume de vendas* & 233486,8 & 260936,9 & 274456,1 \\
\hline Vendas médias por empresa & 0,87 & 0,85 & 0,80 \\
\hline \multicolumn{4}{|c|}{ Valores percentuais dos SIC em relação ao total dos sectores (\%) } \\
\hline Empresas & 11,8 & 16,3 & 18,6 \\
\hline Emprego & 20,8 & 22,7 & 26,0 \\
\hline Vendas & 15,4 & 26,1 & 25,0 \\
\hline
\end{tabular}

Fonte: Quadros de Pessoal e cálculos dos autores.

* Valores em milhões de euros, a preços constantes de 2000. 
Neste estudo designam-se por empresas estrangeiras (EE) aquelas empresas em que mais de $50 \%$ do capital social é detido por investidores estrangeiros; designam-se por empresas participadas (EP) aquelas empresas cuja participação estrangeira no capital social é positiva mas não superior a 50\%; e designam-se por empresas nacionais (EN) aquelas empresas em que $100 \%$ do capital social é detido por investidores nacionais ${ }^{5}$.

\begin{tabular}{|c|c|c|c|c|c|c|}
\hline \multirow[b]{2}{*}{$\mathbf{N}^{\circ}$ empresas (\%) } & \multicolumn{2}{|c|}{2000} & \multicolumn{2}{|c|}{2003} & \multicolumn{2}{|c|}{2006} \\
\hline & Total & SIC & Total & SIC & Total & SIC \\
\hline $\mathrm{EE}$ & 0,8 & 1,1 & 0,9 & 1,3 & 1,0 & 1,4 \\
\hline EP & 0,2 & 0,4 & 0,1 & 0,4 & 0,2 & 0,4 \\
\hline Emprego (\%) & Total & SIC & Total & SIC & Total & SIC \\
\hline EE & 8,4 & 9,7 & 8,4 & 8,9 & 9,0 & 10,8 \\
\hline EP & 2,3 & 3,5 & 2,2 & 4,6 & 2,2 & 4,4 \\
\hline Volume de vendas (\%) & Total & SIC & Total & SIC & Total & SIC \\
\hline EE & 16,5 & 16,1 & 17,9 & 18,7 & 16,9 & 15,8 \\
\hline EP & 6,2 & 9,7 & 6,7 & 13,6 & 7,6 & 17,2 \\
\hline
\end{tabular}

Fonte: Quadros de Pessoal e cálculos dos autores.

EE: empresas estrangeiras (EE) são empresas em que mais de $50 \%$ do capital social é detido por investidores estrangeiros; EP: Empresas participadas são empresas em que a participação estrangeira no capital é positiva mas não superior a $50 \%$.

Como se pode observar na Tabela 2, existe uma maior percentagem de EE e EP nos SIC do que na totalidade dos sectores, tendo-se verificado um ligeiro aumento do peso relativo das empresas de propriedade estrangeira na economia portuguesa no período de 2000 a 2006 . No que respeita ao emprego, também se verificou um aumento do peso do emprego criado pelas empresas de propriedade estrangeira, sobretudo nos SIC. Na verdade, a percentagem do emprego total criado pelas EE nos SIC aumentou de 9,7\% em 2000 para 10,8\% em 2006, e de 3,5\% para 4,4\% nas EP. No que respeita ao peso do volume de vendas, os dados parecem evidenciar uma tendência de crescimento para as EP, quer nos SIC quer na totalidade dos sectores, e uma tendência de estabilização para as EE.

\begin{tabular}{|c|c|c|c|c|c|c|}
\hline \multirow[b]{2}{*}{ Tipo de empresa } & \multicolumn{2}{|c|}{2000} & \multicolumn{2}{|c|}{2003} & \multicolumn{2}{|c|}{2006} \\
\hline & Total & SIC & Total & SIC & Total & SIC \\
\hline $\mathrm{EE}$ & 139,9 & 128,5 & 145,4 & 133,2 & 145,4 & 132,2 \\
\hline EP & 129,0 & 134,1 & 138,3 & 133,3 & 127,8 & 105,3 \\
\hline EN & 59,2 & 47,7 & 56,7 & 47,1 & 55,4 & 47,3 \\
\hline
\end{tabular}

Fonte: Quadros de Pessoal e cálculos dos autores.

*Valores em milhares de euros, a preços constantes de 2000. 
De acordo com os dados constantes na Tabela 3, verificamos também que as empresas estrangeiras são, em média, mais produtivas que as empresas nacionais quando se considera a produtividade medida pelo volume de vendas médio por trabalhador.

\subsection{Modelo Econométrico}

O modelo que se pretende estimar é um modelo em que a variável dependente, y, corresponde a uma proporção, nomeadamente à proporção de trabalhadores da empresa num determinado nível de habilitações/qualificações, como mais à frente iremos definir ${ }^{7}$. A variável dependente irá assumir valores compreendidos entre 0 e 1 , isto é, $0 \leq y_{i} \leq 1$. Nestas circunstâncias, e como referem Papke e Wooldridge (1996), os modelos lineares não são os mais adequados pois padecem das mesmas desvantagens que o modelo linear de probabilidade no caso de variável dependente binária. Ou seja, não está assegurado que os valores estimados de y estejam compreendidos entre 0 e 1 e, por outro lado, não é razoável, assumir que os efeitos de uma dada alteração na variável explicativa $x_{\mathrm{j}}$, sejam constantes no intervalo de valores admissíveis para o vector $\mathbf{x}$ das variáveis explicativas do modelo. Deste modo, os mesmos autores sugerem a utilização de um modelo fractional logit que ultrapassa as dificuldades referidas.

A forma funcional do modelo é a seguinte: $E\left(y / x_{i}\right)=G\left(x_{i} \beta\right)$, em que $G($.$) é a função distribuição logística e, portanto, E\left(y / x_{i}\right)=\exp \left(x_{i} \beta\right) /\left[1+\exp \left(x_{i} \beta\right)\right]$. Para obter as estimativas dos coeficientes de regressão $\beta$, recorrer-se-á ao método da quasi-máxima verosimilhança.

Em particular, a especificação do modelo a estimar é a seguinte:

$\mathrm{E}\left(\mathrm{y}_{\mathrm{it}} / \mathbf{x}_{\mathrm{it}}, \alpha_{\mathrm{i}}\right)=\mathrm{G}\left(\alpha_{\mathrm{i}}+\beta_{1} \mathrm{CSE} 1_{\mathrm{it}}+\beta_{2} \mathrm{CSE} 2_{\mathrm{it}}+\beta_{3} \mathrm{IDADE}_{\mathrm{it}}+\beta_{4} \mathrm{LDIM}_{\mathrm{it}}+\beta_{5 \mathrm{~J}} \sum_{J=1}^{6} R E G J_{i t}+\right.$

$\left.\beta_{6 \mathrm{~J}} \sum_{J=1}^{48} C A E J_{i t}+\beta_{7 \mathrm{~T}} \sum_{T=1}^{5} A N O T_{t}\right)$

em que $i$ representa a i-ésima empresa do sector de SIC, $t$ o período de referencia e $\alpha_{i}$ um efeito fixo empresa específico. Na secção seguinte, procede-se à descrição de cada uma das variáveis incluídas em (1).

\subsection{Variáveis}

\subsubsection{Capital humano}

Por capital humano entende-se o conjunto de competências e conhecimentos que um ou mais trabalhadores detêm e que podem ser usados no processo produtivo. Normalmente estão relacionadas com o nível educacional formal ou com o trabalho e neste caso são desenvolvidas através de formação ou experiência. Neste artigo utilizamos cinco medidas para caracterizar a intensidade do capital humano das empresas e que correspondem às cinco definições da variável dependente. Assim, usou-se a percentagem de trabalhadores da empresa com pelo menos 12 anos de escolaridade (EDU) e a percentagem de licenciados (LIC) da empresa para medir as habilitações escolares. A percentagem de trabalhadores da empresa que pertencem aos quadros superiores (QS), a percentagem de profissionais altamente qualificados (PA) e a percentagem de engenheiros (ENG), foram as proxies utilizadas para medir as qualificações. Níveis mais elevados nestes rácios, significam níveis mais elevados de intensidade de capital humano e, logo, sinónimo de maiores competências profissionais e conhecimentos ${ }^{8}$.

7 Habilitações e qualificações são conceitos que embora utilizados de forma quase indistinta na literatura são diferentes. As qualificações são competências específicas que são adquiridas essencialmente através do learning by doing ou do on-the-job training, enquanto as habilitações traduzem competências genéricas que se adquirem através da educação e da formação formal (Teixeira e Tavares-Lehmann, 2007).

8 Vários autores medem intensidade de capital humano pela percentagem de trabalhadores com determinado 
Os dados reflectidos na Tabela 4 revelam que as empresas do sector dos SIC detêm uma maior percentagem de licenciados e de trabalhadores com pelo menos 12 anos de escolaridade, independentemente da propriedade do capital social. As empresas dos SIC possuem também uma maior percentagem de quadros superiores, de quadros médios e de profissionais altamente qualificados.

Em relação à influência da propriedade do capital, as empresas estrangeiras e com participação estrangeira apresentam uma maior percentagem de trabalhadores com elevada formação do que as empresas nacionais. As empresas estrangeiras possuem também uma maior percentagem de quadros superiores e médios, e de profissionais altamente qualificados. As diferenças são mais significativas quando se considera a totalidade dos sectores. Estes resultados, sem condicionantes, estão de acordo com a hipótese de que as empresas multinacionais empregam trabalhadores com maior nível de habilitações e qualificações (Almeida, 2004).

\begin{tabular}{|c|c|c|c|c|c|c|}
\hline & \multicolumn{3}{|c|}{ SIC } & \multicolumn{3}{|c|}{ Todos os sectores } \\
\hline & EE & EP & EN & EE & EP & EN \\
\hline \multicolumn{7}{|l|}{ Percentagem de trabalhadores } \\
\hline Com licenciatura & 38,8 & 48,2 & 22,6 & 21,6 & 22,6 & 5,9 \\
\hline Com $>=12$ anos escolaridade & 81,5 & 80,9 & 60,5 & 59,2 & 55,3 & 24,6 \\
\hline \multicolumn{7}{|l|}{ Percentagem de trabalhadores } \\
\hline Quadros superiores e médios & 31,9 & 37,4 & 29,1 & 18,9 & 23,1 & 22,1 \\
\hline Altamente qualificados & 16,3 & 15,8 & 9,4 & 13,3 & 10,9 & 4,0 \\
\hline Engenheiros & 6,0 & 6,8 & 2,1 & 4,5 & 5,0 & 0,8 \\
\hline
\end{tabular}

Fonte: Quadros de Pessoal e cálculos dos autores.

\subsubsection{Covariáveis: Propriedade estrangeira, idade, dimensão, localização geográfica e sector de actividade}

A principal variável explicativa do nosso modelo é a propriedade do capital social. Tal como referido atrás, consideramos três categorias: a participação estrangeira no capital social é superior a 50\% (CSE1), a participação estrangeira no capital social é positiva e não superior a $50 \%$ (CSE2), a participação estrangeira no capital social é nula (categoria omitida).

Foram utilizadas as seguintes variáveis de controlo: dimensão (logaritmo do número total de trabalhadores da empresa; LDIM) e idade (número de anos de actividade desde a data da constituição; IDADE) dado que estas podem afectar a política de recrutamento da empresa (Teixeira e Tavares-Lehmann, 2007). Também foram incluídas variáveis dummy para os vários sectores de actividade dos $\operatorname{SIC}^{9}$ (CAE a 3 dígitos) e para a localização geográfica (ao nível das NUTSII), com o intuito de controlar para o facto da intensidade de capital humano poder estar associada com o sector de actividade dentro dos SIC ou com a localização geográfica da empresa.

No que respeita à dimensão da empresa, verifica-se que as empresas estrangeiras e com participação apresentam, em média, uma maior dimensão do que as empresas nacionais (ver Tabela 5). Estas últimas são, na sua grande maioria, empresas de menor dimensão, ou seja, $85 \%$ das empresas nacionais nos SIC têm menos de 10 trabalhadores. Igual percentagem se 
observa quando se considera a totalidade dos sectores. Apenas $0,2 \%$ das empresas nacionais empregavam mais de 250 trabalhadores $(0,5 \%$ para os SIC). Para as empresas estrangeiras e com participação estrangeira esta percentagem eleva-se, respectivamente, para 5,2\% e 6,7\% nos SIC e 6,7\% nos dois casos para o total dos sectores.

No que respeita à idade não se verificam diferenças significativas entre as empresas estrangeiras e as nacionais (ver Tabela 6). Em ambos os casos verificamos que existe uma percentagem significativa de Start-ups, isto é, de empresas com menos de 10 anos de idade ${ }^{10}$, mas que é mais elevada nos SIC do que na totalidade dos sectores. Estes resultados não estão de acordo com os obtidos por Teixeira e Tavares-Lehmann (2007), que encontraram uma percentagem muito menor, cerca de $13 \%$, de start-ups para uma amostra de empresas de base tecnológica para o período de 2001-2003. Esta evidência, aliada ao facto de $68 \%$ das empresas consideradas na amostra utilizada por aquelas autoras empregarem entre 10 e 250 trabalhadores, parece sugerir que na lista de empresas da Markelink de 2004, que inclui todas as empresas localizadas em Portugal que declaram e publicitam actividades de I\&D, há uma clara sub-representação das micro e pequenas empresas e de empresas recém-criadas.

\begin{tabular}{|c|c|c|c|c|c|c|}
\hline & \multicolumn{3}{|c|}{ SIC } & \multicolumn{3}{|c|}{ Todos os sectores } \\
\hline & EE & EP & EN & EE & EP & EN \\
\hline Micro empresas: 1-9 & 53,2 & 52,9 & 85,0 & 41,8 & 44,0 & 84,6 \\
\hline Pequenas: 10-49 & 27,8 & 24,4 & 12,2 & 32,3 & 30,0 & 13,4 \\
\hline Médias pequenas: 50-249 & 13,7 & 16,0 & 2,3 & 19,3 & 19,4 & 1,8 \\
\hline Médias grandes: 250-499 & 2,7 & 2,2 & 0,3 & 3,9 & 3,5 & 0,1 \\
\hline Grandes: >= 500 & 2,5 & 4,5 & 0,2 & 2,8 & 3,2 & 0,1 \\
\hline
\end{tabular}

Fonte: Quadros de Pessoal e cálculos dos autores.

\begin{tabular}{|c|c|c|c|c|c|c|}
\hline & \multicolumn{3}{|c|}{ SIC } & \multicolumn{3}{|c|}{ Todos os sectores } \\
\hline & EE & EP & EN & EE & EP & EN \\
\hline Idade (n. ${ }^{\circ}$ de anos) & 10,80 & 10,97 & 11,11 & 13,60 & 13,80 & 11,73 \\
\hline \multicolumn{7}{|c|}{$\begin{array}{l}\text { Distribuição das empresas } \\
\text { por idade (\%): }\end{array}$} \\
\hline $0-9$ anos & 60,9 & 54,0 & 58,6 & 50,6 & 48,9 & 52,2 \\
\hline 10-19 anos & 23,7 & 31,7 & 23,4 & 27,5 & 29,6 & 25,3 \\
\hline 20-29 anos & 4,8 & 5,9 & 7,1 & 7,2 & 8,0 & 9,2 \\
\hline$>=30$ anos & 7,1 & 5,1 & 6,6 & 14,7 & 13,5 & 13,2 \\
\hline Não definido & 3,5 & 3,3 & 4,3 & 0 & 0 & 0 \\
\hline
\end{tabular}

Fonte: Quadros de Pessoal e cálculos dos autores.

Verificamos que a maioria das empresas estrangeiras e com participação estrangeira se situam na zona de Lisboa (NUTS II): 57,6 \% e 50,6\%, respectivamente, na totalidade dos sectores. Quando se considera os SIC, estes valores elevam-se para 73,8\% e 69,8\% (ver Tabela 7). 


\begin{tabular}{|c|c|c|c|c|c|c|}
\hline & \multicolumn{3}{|c|}{ SIC } & \multicolumn{3}{|c|}{ Todos os sectores } \\
\hline & EE & EP & EN & EE & EP & EN \\
\hline Norte & 10,0 & 18,7 & 29,7 & 21,0 & 26,8 & 35,0 \\
\hline Centro & 2,7 & 5,0 & 19,4 & 10,2 & 13,4 & 22,9 \\
\hline Lisboa & 73,8 & 69,8 & 36,0 & 57,6 & 50,6 & 25,4 \\
\hline Alentejo & 1,3 & 1,8 & 5,6 & 4,4 & 3,8 & 7,5 \\
\hline Algarve & 6,0 & 2,0 & 5,7 & 4,3 & 2,9 & 5,5 \\
\hline Açores & 0,2 & 0,0 & 1,6 & 0,2 & 0,5 & 1,8 \\
\hline Madeira & 6,0 & 2,7 & 2,0 & 2,3 & 2,0 & 2,0 \\
\hline
\end{tabular}

Fonte: Quadros de Pessoal e cálculos dos autores.

Finalmente, quanto à distribuição das empresas estrangeiras por sector de actividade económica, a actividade económica que apresenta uma maior percentagem de empresas estrangeiras e participadas é o "Comércio por grosso e a retalho» (CAE G), seguido pela «Actividade transformadora» (CAE D) e pelas «Actividades imobiliárias» (CAE K). Nos SIC, a maioria das EE e das EP estão no subsector 74 "Outras actividades de serviços prestados principalmente às empresas", e, dentro deste, no subsector 741 "Actividades Jurídicas, Contabilidade e auditoria .... e no subsector 748 "Outras actividades de serviços prestados principalmente às empresas».

\subsection{Resultados empíricos}

O modelo especificado em (1) foi estimado por recurso ao estimador pooled fractional logit ignorando, portanto, a estrutura em painel dos dados. Este estimador é consistente, se assumirmos que a componente não observada, $\alpha_{i}$, não está correlacionada com as variáveis explicativas incluídas no modelo ${ }^{11}$. No intuito de termos em conta que os termos de perturbação do modelo podem exibir correlação temporal para cada empresa $i$ e, para efeitos de comparação, usou-se ainda o estimador fractional logit com efeitos aleatórios. Este modelo assume que $\alpha_{i}$ segue uma distribuição normal de média nula e variância constante, ou seja, $\alpha_{i} \sim \mathrm{N}\left(0, \sigma^{2}\right)$. Neste caso, no entanto, $\operatorname{Pr}\left(\mathrm{y}_{\mathrm{it}}=1 \mid \mathrm{x}_{i t}, \beta\right) \neq \Lambda\left(\mathrm{x}_{i t} \beta\right)$, pelo que os parâmetros do modelo não são directamente comparáveis com os do modelo pooled fractional logit. Na verdade, aquela probabilidade depende do parâmetro desconhecido, $\alpha_{i}$, que o modelo não estima (Cameron and Trivedi, 2009).

Os resultados obtidos são apresentados na Tabela 8 para os SIC e total dos sectores. Os resultados obtidos pelo modelo pooled fractional logit e pelo modelo fractional logit com efeitos aleatórios não são, em geral, qualitativamente diferentes. Com excepção da variável dimensão, os efeitos estimados associados a cada uma das variáveis explicativas apresentam o mesmo sinal em qualquer dos modelos e são estatisticamente significativos. Em particular, verifica-se que, para todas as medidas utilizadas para a variável dependente, as empresas com participação estrangeira no capital social apresentam, tudo o resto constante, uma maior intensidade de capital humano (SIC e total dos sectores) do que as empresas nacionais.

$11 \mathrm{Em}$ alternativa, seria de todo o interesse abandonar este pressuposto, e usar o estimador de efeitos fixos que permitiria controlar para a heterogeneidade individual (permanente) não observada das empresas. No entanto, este método não permite identificar os efeitos das variáveis explicativas que são constantes ao longo do tempo. Como no nosso caso, as principais variáveis de interesse - CSE1 e CSE2 - são constantes no período considerado para a generalidade das empresas, não será possível identificar os seus efeitos se usarmos um modelo logit com efeitos fixos. De qualquer modo, as consequências resultantes de não se controlar para a heterogeneidade individual (permanente) não observada das empresas estão, de certo modo, minimizadas, devido à diversidade de informação utilizada para caracterizar as empresas. 


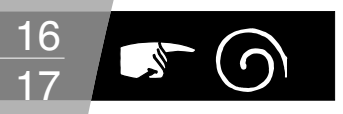

\begin{tabular}{|c|c|c|c|c|c|c|c|c|c|c|}
\hline \multicolumn{6}{|c|}{ pooled fractional logit } & \multicolumn{5}{|c|}{ fractional logit com efeitos aleatórios } \\
\hline & LIC & EDU & QS & PA & ENG & LIC & EDU & QS & PA & ENG \\
\hline \multicolumn{6}{|c|}{ SIC (N = 301823) } & \multicolumn{5}{|c|}{ SIC (N = 301823) } \\
\hline \multirow[t]{2}{*}{ CSE1 } & $0,7501^{*}$ & $0,8381^{*}$ & $0,1570^{*}$ & $0,3450^{*}$ & $0,6229^{*}$ & $0,3791^{*}$ & $0,4297^{\star}$ & $0,1005^{\star *}$ & $0,2736^{*}$ & $0,4097^{*}$ \\
\hline & $(0,0412)$ & $(0,0482)$ & $(0,0389)$ & $(0,0456)$ & $(0,0831)$ & $(0,0344)$ & $(0,0341)$ & $(0,0652)$ & $(0,0450)$ & $(0,0893)$ \\
\hline \multirow[t]{2}{*}{ CSE2 } & $0,9246^{\star}$ & $0,8238^{*}$ & $0,4701^{*}$ & $0,2817^{*}$ & $0,7722^{*}$ & $0,4325^{*}$ & $0,3941^{*}$ & $0,2384^{*}$ & $0,1785^{\star \star}$ & $0,4306^{\star *}$ \\
\hline & $(0,0792)$ & $(0,0895)$ & $(0,072)$ & $(0,0695)$ & $(0,1394)$ & $(0,0587)$ & $(0,0514)$ & $(0,0343)$ & $(0,0708)$ & $(0,1613)$ \\
\hline \multirow[t]{2}{*}{ IDADE } & $-0,0265^{\star}$ & $-0,0187^{\star}$ & $-0,0333^{*}$ & $0,0040^{*}$ & $-0,0236^{\star}$ & $-0,0253^{*}$ & $-0,0178^{\star}$ & $-0,0349^{*}$ & $0,0033^{*}$ & $-0,0259^{*}$ \\
\hline & $(0,0008)$ & $(0,0006)$ & $(0,0008)$ & $(0,0006)$ & $(0,0024)$ & $(0,0008)$ & $(0,0005)$ & $(0,0007)$ & $(0,0006)$ & $(0,0024)$ \\
\hline \multirow[t]{2}{*}{ LDIM } & $0,0526^{*}$ & $0,0297^{\star}$ & $-0,2544^{\star}$ & $0,1071^{*}$ & $0,0413^{*}$ & $0,0101^{* * *}$ & $-0,0276^{\star}$ & $-0,3087^{\star}$ & $0,1019^{*}$ & $-0,0136$ \\
\hline & $(0,0062)$ & $(0,0053)$ & $(0,0054)$ & $(0,0067)$ & $(0,0152)$ & $(0,0060)$ & $(0,0050)$ & $(0,0055)$ & $(0,0066)$ & $(0,0156)$ \\
\hline \multirow[t]{2}{*}{ Constante } & $-2,6337$ & $-0,6623$ & $-0,2574$ & $-3,2451$ & $-5,6025$ & $-2,2426$ & $-0,4013$ & $-0,1934$ & $-3,1248$ & $-5,0714$ \\
\hline & $(0,0892)$ & $(0,0544)$ & $(0,0528)$ & $(0,0938)$ & $(0,2975)$ & $(0,0760)$ & $(0,0435)$ & $(0,0469)$ & $(0,0873)$ & $(0,2583)$ \\
\hline Teste-Wald & 14513,80 & 21365,79 & 16682,63 & 7925,46 & 7211,99 & 11045,71 & 18238,27 & 15859,70 & 6469,40 & 4916,05 \\
\hline$p$-value & 0,0000 & 0,0000 & 0,0000 & 0,0000 & 0,0000 & 0,0000 & 0,0000 & 0,0000 & 0,0000 & 0,0000 \\
\hline \multicolumn{6}{|c|}{ Efeitos marginais } & \multicolumn{5}{|c|}{ Efeitos marginais } \\
\hline CSE1 & $0,1345^{*}$ & $0,1826^{*}$ & $0,0320^{*}$ & $0,0307^{\star}$ & $0,0083^{*}$ & $0,0629^{*}$ & $0,1006^{*}$ & $0,0201^{*}$ & $0,0236^{*}$ & $0,0053^{*}$ \\
\hline \multirow[t]{2}{*}{ CSE2 } & $0,1735^{\star}$ & $0,1795^{*}$ & $0,1017^{\star}$ & $0,0245^{*}$ & $0,0112^{*}$ & $0,0730^{\star}$ & $0,0923^{*}$ & $0,0490^{* *}$ & $0,0150^{* *}$ & $0,0057^{\star *}$ \\
\hline & \multicolumn{5}{|c|}{ Todos os sectores ( $\mathrm{N}=1836765)$} & \multicolumn{5}{|c|}{ Todos os sectores $(\mathrm{N}=1836765)$} \\
\hline \multirow[t]{2}{*}{ CSE1 } & $1,1089^{\star}$ & $1,2651^{*}$ & $0,0375^{\star \star \star}$ & $0,8356^{\star}$ & $1,1394^{*}$ & $0,5719^{\star}$ & $0,5731^{*}$ & $0,0652^{*}$ & $0,5600^{*}$ & $0,7464^{*}$ \\
\hline & $(0,0253)$ & $(0,0211)$ & $(0,0200)$ & $(0,0264)$ & $(0,0472)$ & $(0,0486)$ & $(0,0166)$ & $(0,0183)$ & $(0,0298)$ & $(0,0611)$ \\
\hline \multirow[t]{2}{*}{ CSE2 } & $1,0938^{\star}$ & $1,0804^{*}$ & $0,2968^{*}$ & $0,5715^{*}$ & $1,1047^{*}$ & $0,4781^{*}$ & $0,4123^{*}$ & $0,1439^{*}$ & $0,3677^{\star}$ & $0,6290^{*}$ \\
\hline & $(0,0505)$ & $(0,0419)$ & $(0,0398)$ & $(0,0497)$ & $(0,0878)$ & $(0,0270)$ & $(0,0284)$ & $(0,0369)$ & $(0,0541)$ & $(0,1327)$ \\
\hline \multirow[t]{2}{*}{ IDADE } & $-0,0135^{\star}$ & $-0,0140^{*}$ & $-0,0190^{*}$ & $0,0059^{*}$ & $-0,0132^{*}$ & $-0,0143^{*}$ & $-0,0134^{*}$ & $-0,0191^{*}$ & $0,0050^{*}$ & $-0,0139^{*}$ \\
\hline & $(0,0004)$ & $(0,0003)$ & $(0,0003)$ & $(0,0004)$ & $(0,0009)$ & $(0,0006)$ & $(0,0003)$ & $(0,0003)$ & $(0,0004)$ & $(0,0009)$ \\
\hline \multirow[t]{2}{*}{ LDIM } & $0,1041^{*}$ & $0,0688^{*}$ & $-0,3035^{\star}$ & $0,1538^{*}$ & $0,1465^{*}$ & $0,0198^{*}$ & $-0,0177^{\star}$ & $-0,3566^{*}$ & $0,1399^{*}$ & $0,0572^{*}$ \\
\hline & $(0,0038)$ & $(0,0025)$ & $(0,0022)$ & $(0,0037)$ & $(0,0079)$ & $(0,0040)$ & $(0,0024)$ & $(0,0024)$ & $(0,0039)$ & $0,0092)$ \\
\hline \multirow[t]{2}{*}{ Constante } & $-3,1523$ & $-1,4544$ & $-0,5252$ & $-3,6280$ & $-5,2690$ & $-2,7723$ & $-1,0965$ & $-0,4137$ & $-3,4480$ & $-5,0622$ \\
\hline & $(0,0117)$ & $(0,0007)$ & $(0,0067)$ & $(0,0128)$ & $(0,0300)$ & $(0,0117)$ & $(0,0007)$ & $(0,0067)$ & $(0,0128)$ & $(0,0300)$ \\
\hline Teste-Wald & 71352,71 & 112855,97 & 69313,62 & 29783,50 & 15770,72 & 43833,25 & 72822,62 & 73029,29 & 16920,79 & 8689,74 \\
\hline $\mathrm{p}$-value & 0,0000 & 0,0000 & 0,0000 & 0,0000 & 0,0000 & 0,0000 & 0,0000 & 0,0000 & 0,0000 & 0,0000 \\
\hline \multicolumn{6}{|c|}{ Efeitos marginais } & \multicolumn{5}{|c|}{ Efeitos marginais } \\
\hline CSE1 & $0,0804^{*}$ & $0,2827^{\star}$ & $0,0062^{\star \star \star}$ & $0,0415^{*}$ & $0,0128^{*}$ & $0,0344^{*}$ & $0,1166^{*}$ & $0,0106^{\star}$ & $0,0247^{\star}$ & $0,0070^{*}$ \\
\hline CSE2 & $0,0793^{\star}$ & $0,2373^{*}$ & $0,0530^{*}$ & $0,0251^{*}$ & $0,0123^{*}$ & $0,0276^{\star}$ & $0,0812^{*}$ & $0,0240^{*}$ & $0,0148^{\star}$ & $0,0055^{\star}$ \\
\hline
\end{tabular}

Fonte: A especificação inclui ainda dummies temporais, sectoriais e regionais (NUTS II). Os valores que aparecem na primeira linha são as estimativas dos coeficientes e os que aparecem entre parêntesis são os desvios padrões robustos à presença de correlação temporal nos erros aleatórios para cada empresa i. Os símbolos *, ** $\mathrm{e}^{* \star *}$ significam que as variáveis são estatisticamente significativas a $1 \%, 5 \%$ e $10 \%$, respectivamente. 
Considerando o modelo pooled fractional logit, e de acordo com as estimativas obtidas para os efeitos marginais estima-se que, em média, as empresas com mais de $50 \%$ de capital estrangeiro apresentem uma percentagem de licenciados (LIC) superior a uma empresa nacional em 13,45 pontos percentuais (p. p.), mantendo-se tudo o resto constante ${ }^{12}$. Quando a variável dependente é medida pela percentagem de trabalhadores com pelo menos 12 anos de escolaridade (EDU) este acréscimo é de 18,26 p. p., mantendo-se tudo o resto constante. Para as qualificações este acréscimo é menor: 3,20 p. p. para a percentagem de quadros superiores (QS), 3,07 p. p. para os profissionais altamente qualificados (PA) e apenas 0,83 p. p. para a percentagem de engenheiros (ENG). Estes resultados são consistentes com os obtidos por Teixeira e Tavares-Lehmann (2007), que evidenciaram que o impacto da propriedade estrangeira sobre a intensidade de capital humano é maior quando se considera uma medida do capital humano geral como a escolaridade do que quando se utiliza uma medida do capital humano específico como as qualificações ${ }^{13}$.

Para as empresas cuja participação estrangeira não excede os $50 \%$ do capital social estima-se que, em média, apresentem um acréscimo de 17,35 p. p. na percentagem de licenciados face às empresas nacionais, mantendo-se tudo o resto constante. Quando a variável dependente é EDU este acréscimo é de 17,95 p. p., para os QS é de 10,17 p. p., 2,45 p. p. para a percentagem de PA e apenas 1,12 p. p. para a percentagem de ENG, mantendo-se tudo o resto constante. Estes resultados parecem sugerir que o impacto de ter propriedade estrangeira sobre a intensidade do capital humano é relativamente independente do montante da participação estrangeira no capital social da empresa.

A idade tem um efeito negativo na intensidade do capital humano (à excepção dos PA), isto é, as empresas mais novas apresentam uma maior intensidade de capital humano. Este resultado não é inesperado, visto que, em geral, as empresas mais velhas possuem uma força de trabalho com mais antiguidade ou idade e, portanto, com menos habilitações escolares. Até que possa ocorrer uma substituição da força de trabalho actual por outra mais qualificada vai demorar algumas gerações.

A dimensão tem um efeito positivo (à excepção dos QS), isto é, as empresas de maior dimensão tenderão a contratar uma força de trabalho mais qualificada e escolarizada ${ }^{14}$. Estes resultados contradizem os obtidos por Teixeira e Tavares-Lehmann (2007) que obtiveram um efeito negativo e estatisticamente significativo para a variável dimensão. Este impacto positivo da dimensão poderá ser explicado por vários motivos, nomeadamente, a existência de mercados de trabalho internos que tornam estas empresas mais atractivas sob o ponto de vista dos trabalhadores mais qualificados ou escolarizados e que têm maiores possibilidades de progressão na carreira.

Por último, vale a pena referir que as empresas com sede na região de Lisboa tendem a ter uma maior intensidade de capital humano. Uma possível explicação poderá ter a ver com as diferentes restrições em termos de oferta de trabalho que as várias regiões enfrentam.

Em síntese, verifica-se que, para todas as medidas utilizadas para a variável dependente, as empresas com participação estrangeira no capital social apresentam, tudo o resto constante, uma maior intensidade de capital humano (SIC e total dos sectores) do que as empresas nacionais. Também se constata que o efeito de ter propriedade estrangeira é superior quando $o$ capital humano é medido pelas habilitações escolares do que quando é medido pelas qualificações. 
Por outro lado, os resultados também parecem sugerir que a maior intensidade de capital humano é independente da percentagem que os investidores estrangeiros possuem no capital social da empresa.

\section{Conclusão}

A análise incide sobre a economia portuguesa, país que tem implementado uma política activa de desenvolvimento dos SIC e simultaneamente de atracção de IDE, apresentando no entanto elevados défices de qualificações (Bernardino et al., 2010; Teixeira e Tavares-Lehmann, 2007). O estudo apoia-se na base de dados dos Quadros de Pessoal, extremamente representativa do sector privado da economia portuguesa. $\mathrm{O}$ trabalho analisa o sector dos serviços dada a sua importância em termos de output, emprego e investimento estrangeiro. De acordo com o que julgamos saber, é o primeiro estudo nesta temática que analisa, em termos absolutos e comparativos face aos restantes sectores, os serviços intensivos em conhecimento, recorrendo a uma base de dados representativa das empresas portuguesas no sector privado da economia adoptando uma metodologia econométrica adequada.

Os resultados empíricos confirmam que as empresas multinacionais apresentam uma maior intensidade de capital humano, embora não exista evidência de uma grande heterogeneidade associada ao grau de participação estrangeira no capital social. Estes resultados são válidos para o sub-sector dos serviços intensivos em conhecimento, bem como para a totalidade dos sectores.

Neste sentido, Portugal deve seguir uma política de atracção de IDE intensivo em conhecimento e ao mesmo tempo uma política de recursos humanos atenta ao tipo de competências que é necessário desenvolver. Um stock de capital humano leva anos a construir (Teixeira e TavaresLehmann, 2007) pelo que se torna fundamental uma política de educação e formação profissional que não dependa do ciclo político. É necessário que a sociedade globalmente tenha consciência da importância do capital humano como elemento gerador de riqueza a longo prazo.

Como sugestão de pesquisa futura, seria interessante analisar se as empresas multinacionais têm também um comportamento diferenciador em matéria de formação profissional. Qual a natureza das competências transmitidas pelo IDE e, de que forma, as instituições de formação (escolas de negócios, por exemplo) poderão interagir mais com as empresas estrangeiras (e nacionais) e complementar o learning-by-doing (Blomström e Kokko, 2003). 
Almeida, Rita (2004) The Labour Market Effects of Foreign Owned Firms, Journal of International Economics, 72, 75-96.

Baldwin, Richard; Braconier, Henry e Forglid, Rikard (2005) Multinationals, Endogenous Growth and Technological Spillovers, Theory and Evidence, Review of International Economics, 13, 945$-963$.

Barbosa, Natália (2007) Implicações da Mudança de Propriedade na Composição da Força de Trabalho, Cadernos Sociedade e Trabalho, 9, 85-92.

Bergman, Annika (2006) FDI and Spillover Effects in the Indian Pharmaceutical Industry, RIS Discussion Paper No. 113.

Bernardino, L., Jones, M.V. And Morgan-Thomas, A. (2010) Resources and Performance in Foreign markets: The Case of High-Technology SMEs in Portugal, em Dimitratos, Pavlos e Jones, M.V. (eds.) Resources, Efficiency and Globalization, London: Palgrave MacMillan, 249-267.

Bishop, Paul (2008) Spatial Spillovers and the Growth of Knowledge Intensive Services, Tijdschrift voor economische en sociale geografie (Royal Dutch Geographical Society KNAG), 99, 3, 281-292.

Blomström, Magnus e Kokko, Ari (1998) Multinationals Corporations, and Spillovers, Journal of Economic Surveys, 12, 247-277.

Blomström, Magnus e Kokko, Ari (2003) Human Capital and Inward FDI, CEPR Discussion Paper No. 3762.

Boddewyn, J. J., Halbrich, Marsha, B. e Perry, A.C. (1986) Service Multinationals: Conceptualization, Measurement and Theory, Journal of International Business Studies, 17, 41$-57$.

Bouquet, Cyril, Hebert, Louise e Délios, Andrew (2004) Foreign Expansion in Service Industries: Separability and Human Capital Intensity, Journal of Business Research, 57, 35-46.

Cameron, A. Colin e Trivedi, Pravin K. (2009) Microeconometrics Using STATA. Texas: Stata Press, College Station.

Dunning, John H. (1977) Trade, Location of Economic Activity and the MNE: A Search for an Eclectic Approach, em Ohlin, B., Hesselborn, P.O. e Wijkman, P.M. (eds.) The International Allocation of Economic Activity, London: Macmillan.

Dunning, John H. (1989) Trade and Foreign-owned production in Services: some conceptual and theoretical issues, em Giersch, Herbert (ed.) Services in World Economic Growth: Symposium 1989, London: West view Press.

European Foundation for the Improvement of Living and Working Conditions (2005) Sector Futures: the Knowledge-intensive Business Services Sector. Disponível em http://www. eurofound.europa.eu/emcc/publications/2005/ef0559en.pdf (acesso em 13 de Março 2010).

EUROSTAT (2004) Business Services: An Analysis of Foreign Affiliates and Business Demography Statistics - Data 2001, Luxembourg: Office for Official Publications of the European Communities.

EUROSTAT (2007) Employment and Earnings in High-Tech Sectors, Statistics in Focus: Science and Technology, 32/2007. Disponível em http://epp.eurostat.ec.europa.eu/cache/ITY_ OFFPUB/KS-SF-07-032/EN/KS-SF-07-032-EN.PDF (acesso em 13 de Maço 2010) 
Harrington, James W. e Daniels, Peter W. (eds.) (2006) Knowledge-based Services,

Internationalization and Regional Development (The Dynamics of Economic Space), Ashgate, Chapter 1, 1-14.

Hymer, Stephen H (1976) The International Operations of National Firms: A Study of Foreign Direct Investment, Cambridge, MA: MIT Press.

Kapstein, Ethan B. (2002) Virtuous circles? Human Capital Formation Economic Development and the Multinational Enterprise, OECD Development Centre Working Paper No. 191.

Larudee, Mehrene e Koechlin, T. (1999) Wages Productivity and Foreign Direct Investment Flows, Journal of Economic Issues, 33, 419-426.

Lipsey, Robert (2004) The Labour Effects of US FDI in Developing Countries, International Labour Office Employment Strategy Paper 2004/6.

Lunati, Maria R. e O'Connor, David (1999) Economic Opening and the Demand for Skills in Developing Countries: a Review of Theory and Evidence, OECD Development Centre, Working Paper No.149.

Miles, I. (2005) Knowledge-intensive-services and innovation, in Bryson, J., Daniels, P. (eds.) The Handbook of Service Industries, Aldershot: Edward Elgar, Chapter 16.

Miles, Ian, Kastrinos Akikos e Glanagan, Kieron (1996) Knowledge - Intensive Services. Users, Carriers and Source of Innovation, EIMS Publicação No.15, Luxemburgo.

Narula, Rajneesh e Marin, Anabel (2003) FDI Spillovers, Absorptive Capacities and Human Capital Development: Evidence from Argentina, MERIT Infonomics Research Memorandum Series Working Paper No. 2003-016.

OECD (2006) Innovation and knowledge Intensive Service Activities, Paris: OECD.

Papke, Leslie E. e Wooldridge, Jeffrey M. (1996) Econometric Methods for Fractional Response Variables, with an Application to 401 (K) Plan Participate Rates, Journal of Applied Econometrics, 11, 619-632.

Quevedo, Garcia J. e Mas-Verdú, Francisco (2008) Does Only the Size Matter in the Use of Knowledge Intensive Services?, Small Business Economics, 31, 137-146.

Siegel, Donald S., Simons, Kenneth L. e Linstrom, Tomas (2005) Ownership Change, Productivity and Human Capital: New Evidence from Matched Employer - Employee Data in Swedish Manufacturing. Artigo apresentado na conferência Producer Dynamics: New Evidence from Micro Data, NBER/CRIW Bethesda; MD.

Slaughter, Mathew J. (2002) Skill Upgrading in Developing Countries: Has Inward FDI played a Role?, OECD Development Center Working Papers No.192.

Slaughter, Mathew J. e Blonigen, A. B. (2001) Foreign Affiliate and U.S: Skill upgrading, The Review of Economics and Statistics, 83, 362-376.

Te Velde, Dirk W. e Xenogiani, Theodora (2007) FDI and International Skill Inequality, Oxford Development Studies, 35, 83-104.

Te Velde, Dirk W. (2005) Globalization and Education, What do the Trade, Investment and Migration Literatures Tell Us?, Overseas Development Institute Working Paper No. 254.

Te Velde, Dirk W. e Morrisey, Oliver (2004) Foreign Direct Investment, Skills and Wage Inequality in East Asia, Journal of the Asia Pacific Economy, 9, 348-369.

Te Velde, Dirk W. e Morrissey, Oliver (2001) Foreign Ownership and Wages: Evidence from Five African Countries, Centre for Research in Economic Development and International Trade University of Nottingham Credit Paper No. 01/19. 
Teixeira, Aurora A. C. e Tavares-Lehmann, Ana T. (2007) Investimento Directo Estrangeiro, Capital Humano e Inovação. Uma aplicação ao caso português, Lisboa: Vida Económica.

Tomlinson, Mark (2002) A New Role for Business Services in Economic Growth, in Archibugi, Daniele e Lundvall, Bengt-Åke (eds.) The Globalizing Learning Economy, Oxford University Press, 97-109.

UNCTAD (2004) The World Investment Report: The Shift Towards Services, New York e Geneva: United Nations.

UNCTAD (2009) World Investment Report 2009 Transnational Corporations, Agricultural Production and Development, New York e Geneva: United Nations.

Wang, Chengqi, Siler, Pamela and Liu, Xiaming (2002) The Relative Economic Performance of Foreign Subsidiaries in UK Manufacturing, Applied Economics, 34, 15, 1885-1892.

Zheng, Connie, Hyland P. e Soosay, C. (2007) Training Practices of Multinational Companies in Asia, Journal of European Industrial Training, 31, 472-494. 